12 scores were analysed using an analysis of covariance model. The IBS-QOL response rates (ie, patients with $\geq 10$-point and $\geq 14$-point increase) for the treatment groups were compared using CochranMantel-Haenszel stratified by geographical region.

Results The changes from baseline in the IBS-QOL "overall" score and seven of the eight subscale scores (Dysphoria, Body Image, Health Worry, Food Avoidance, Social Reaction, Sexual and Relationships) were statistically significant for linaclotide-treated patients vs placebo-treated patients $(p<0.0001$ for each comparison). The percentage of responders for the IBS-QOL "overall" score was statistically significantly greater for linaclotide-treated patients vs placebo-treated patients at week 12 (64.3\% linaclotide-treated patients vs $52.6 \%$ placebo-treated patients for $\geq 10$-point change; $53.8 \%$ linaclotide-treated patients vs $39.1 \%$ placebo-treated patients for $\geq 14$-point change). The most common adverse event among linaclotide-treated patients was diarrhoea.

Conclusion Compared with placebo, once-daily linaclotide treatment for 12 weeks significantly improved "overall" QOL scores and seven out of eight important QOL domains, as measured by the IBS-QOL, in adults with IBS-C.

Competing interests R T Carson Employee of: Forest Research Institute, S Tourkodimitris Employee of: Forest Research Institute, B E Lewis Employee of: Ironwood Pharmaceuticals, J M Johnston Employee of: Ironwood Pharmaceuticals.

\section{PWE-128 REVIEW OF SEHCAT USE AT ST. GEORGE'S 2005-2010: AN UNDERUTILISED INVESTIGATION?}

doi:10.1136/gutjnl-2012-302514d.128

R Holmes, ${ }^{*}$ J 0 Hayat, A Irwin, S Heenan, J-Y Kang, A Poullis. St. George's Hospital, NHS Trust, London, UK

Introduction Bile acid malabsorption (BAM) is a frequently overlooked but easily treatable cause of chronic diarrhoea. The SeHCAT study is a simple non-invasive technique for diagnosing this condition. Three types of BAM are described. Type 1 is seen in patients with terminal ileal disease/resection or bypass. Type 2, known as primary or idiopathic BAM, is characterised by lack of discernable change in ileal histology or obvious clinical history or pathology to account for the malabsorption. Type 3 comprises all other causes of BAM including gastric surgery, pancreatitis, cholecystectomy or associated with microscopic colitis, coeliac disease, diabetes and small bowel bacterial overgrowth.

Methods Retrospective review of all SeHCAT studies performed between 2005 and 2010 at St George's Hospital.

Results Between 1 January 2005 and 31 December 201055 SeHCAT studies were performed. Basic details were available on all 55, however only 44 sets of notes were available. $36(65 \%)$ patients were female and 19 were male. Age ranged from 19 to 77 years old. $62 \%$ of studies were abnormal showing $<15 \%$ retention at 7 days. Of these $11(32 \%)$ demonstrated mild BAM, 8 (24\%) moderate BAM and 15 (44\%) severe BAM. Of the 34 patients with BAM 28 sets of notes were available. 10 (36\%) had Type 1, 8 (29\%) had Type 2 and 10 (36\%) had Type 3 BAM. In those with proven BAM 46\% underwent a trial of bile acid sequestrant (BAS). $88 \%$ of patients with follow-up details had good resolution of their symptoms. Response rates to treatment ranged between 60 and $100 \%$. Six of the 10 type 1 BAM subjects had a trial of BAS; follow-up details are only available on 3, 2 of whom had noticed an improvement in symptoms (66\%). Six of the 8 type 2 BAM subjects had a BAS, follow-up details are available on 5,3 of whom had improvement of their symptoms (60\%). Four of the 10 type 3 BAM subjects had a BAS, at follow-up details are only available on three all of whom had a good response $(100 \%)$.

Conclusion As chronic diarrhoea is a common reason for GI referral, the small number of studies performed over a 5 -year period suggests that SeHCAT is probably underused and bile acid malabsorption under diagnosed. As bile acid sequestrants provide good symptomatic relief, bile acid malabsorption is a useful diagnosis to make.

Competing interests None declared.

\section{PWE-129 SHOULD SEHCAT BE EARLIER IN THE ALGORITHM FOR INVESTIGATING CHRONIC DIARRHOEA?}

doi:10.1136/gutjnl-2012-302514d.129

1R Tunney, * 2J McLaughlin. 'Manchester Medical School, University of Manchester, Salford, UK; ${ }^{2}$ School of Translational Medicine, Manchester University, Manchester, UK

Introduction Bile acid malabsorption (BAM) is a potentially underrecognised cause of chronic diarrhoea. An accurate diagnostic technology exists in the form of the SeHCAT $\left({ }^{75} \mathrm{Se}\right.$-homotaurocholate) test. The British Society of Gastroenterology investigation algorithm places SeHCAT as a very late stage investigation, but if BAM is common or SeHCAT unavailable it may easily be overlooked. This is a treatable condition: the response to bile sequestrants as an empirical "investigation" is not an adequate approach. This study aimed to characterise the results of SeHCAT in a large cohort of patients, and also to determine how well adhered to were the British Society of Gastroenterology guidelines for the investigation of chronic diarrhoea.

Methods The electronic records of 276 patients who underwent SeHCAT scanning between 2005 and 2011 were retrospectively analysed as a medical student project.

Results Bile acid malabsorption (BAM) was very common in patients who underwent SeHCAT testing, found in 110 (40\%) of the 276 patients. In the overall cohort, 136 patients had no prior underlying disease or surgery recorded that might cause diarrhoea, and 86 of these displayed no abnormalities on full screening including endoscopies and coeliac tests. Of the 110 with BAM, 28 had undergone neither endoscopy nor coeliac screening. Predictably, 22 of the 26 (85\%) Crohn's patients with a history of ileal resection had positive results, as did 15 of the 21 (71\%) ileally resected patients who did not have Crohn's disease. Sixteen patients $(55 \%)$ with post-cholecystectomy diarrhoea showed evidence of BAM.

Conclusion BAM is common in subjects undergoing SeHCAT. The current guidelines should be revised to take into account BAM as an important and common cause of diarrhoea, with SeHCAT earlier in the process. It is apparent that clinicians are not widely using the BSG algorithm, presumably using clinical judgement in patients in whom BAM seems likely, and, for example, opting not to undertake colonoscopy in many cases. In the absence of a terminal ileum, the BAM diagnosis is almost universal and the test is probably superfluous. A broader and contemporary health technology assessment including all patients currently investigated for chronic diarrhoea is now required in order to define a new algorithm.

Competing interests None declared.

\section{PWE-130 THE HUMAN GUT MUCOSAL COGNATE CELLULAR RESPONSE TO LIVE ORAL TYPHOID VACCINATION}

doi:10.1136/gutjnl-2012-302514d.130

${ }^{1} S$ Pennington, ${ }^{*}$ A Thompson, ${ }^{1} \mathrm{~A} K \mathrm{~K}$ Wright, ${ }^{1} \mathrm{D}$ Ferreira, ${ }^{1} \mathrm{~A} D \mathrm{D}$ Wright, ${ }^{1} \mathrm{~B}$ Faragher, ${ }^{1} \mathrm{~S}$ B Gordon, ${ }^{2} \mathrm{M}$ A Gordon. ${ }^{1}$ Mucosal Immunology Group, Liverpool School of Tropical Medicine, Liverpool, UK; ${ }^{2}$ Department of Gastroenterology, University of Liverpool, Liverpool, UK

Introduction The human gut mucosal cellular response to oral vaccination has never been directly assessed. We studied the cognate cellular immune response to the live oral typhoid Ty21a vaccine in the gut mucosa of human volunteers, and compared it with that seen in peripheral blood. 